\title{
A novel approach to visual rendering of astro-photographs
}

\author{
Marini D.L.R. , Bonanomi C., Rizzi A. \\ Università degli Studi di Milano, Dipartimento di Informatica \\ Via Comelico 39, 20135, Milano (Italy)
}

\begin{abstract}
When we perform a visual analysis of a cosmic object photograph the contrast plays a fundamental role. A linear distribution of the observable values is not necessarily the best possible for the Human Visual System (HVS). In fact HVS has a non-linear response, and exploits contrast locally with different stretching for different lightness areas. As a consequence, according to the observation task, local contrast can be adjusted to make easier the detection of relevant information. The proposed approach is based on Spatial Color Algorithms (SCA) that mimic the HVS behavior. These algorithms compute each pixel value by a spatial comparison with all (or a subset of) the other pixels of the image. The comparison can be implemented as a weighted difference or as a ratio-product over given sampling in the neighbor region. A final mapping allows exploiting all the available dynamic range. In the case of color images SCA process separately the three chromatic channels producing an effect of color normalization, without introducing channel cross correlation. We will present very promising results on amateur photographs of deep sky objects. The results are presented for a qualitative and subjective visual evaluation and for a quantitative evaluation through image quality measures, in particular to quantify the effect of algorithms on the noise. Moreover our results help to better characterize contrast measures.
\end{abstract}

Keywords: Astrophotography, Contrast image enhancement, Spatial Color Algorithms, Retinex, Contrast measures

\section{INTRODUCTION}

Contrast is essential when performing a visual analysis of cosmic object photograph. A linear distribution of the observable values is not necessarily the best possible for the Human Visual System (HVS). In fact HVS has not a linear response, and exploits contrast locally with different stretching for different lightness areas. Therefore, according to the observation task, it is possible to adjust the local contrast to make easier the detection of relevant information. The information collected by a camera sensor is fundamentally light energy distributed in a spectral interval limited and weighted by the chosen filters. This work proposes a novel approach to render physically meaningful data to make them easily observable. Final results of rendering can be presented on many different devices like monitor, printer or projector. Regardless the fact that each device can be carefully calibrated, each one retains its own characteristic transfer function and consequently potential differences in the resulting appearance.

To render an astro-photograph professional or amateur astronomers normally use Photoshop or similar image editing programs. To solve the tone mapping task of the original picture many cook-books have been proposed that are mainly based on histogram manipulation and masking techniques to modify local contrast, brightness and color. Such methods can be effective but they need a time-consuming trial and error work and the results are cumbersome to reproduce.

The proposed approach is based on Spatial Color Algorithms (SCA) that mimic the HVS behavior. These algorithms compute each pixel value by a spatial comparison with all (or a subset of) the other pixels of the image. The comparison can be implemented as a weighted difference or as a ratio-product over given sampling in the neighbor region. A final mapping allows us to use all the available dynamic range. In the case of color images SCA process separately the three chromatic channels producing an effect of color normalization, without introducing cross correlation between channels. This is an important property for many needs e.g. in emission nebulae analysis.

SCA are unsupervised, they have parameters that can be tuned for specific needs, and they have shown an interesting characteristic: since local contrast is adjusted mimicking HVS mechanisms the resulting images have increased visual information content. Our approach does not exclude the use of other imaging tools for pre and/or post processing needs.

\footnotetext{
*daniele.marini@unimi.it
} 
The drawback of these algorithms is a potential amplification (or addition) of the noise that has not been completely canceled in the captured images and the long computation time.

We will present very promising results on some amateur photographs of deep sky objects. The results are presented for a qualitative and subjective visual evaluation and for a quantitative evaluation through some measures, in particular to quantify the effect of algorithms on the noise.

To characterize the effect on contrast improvement of different post processing algorithms we have also applied some frequently used measures. A comparison of their ability to quantify the contrast improvement shows that many of them are not effective. We also highlight that visual inspection can still provide a final subjective conclusion.

In paragraph 2 we review some image quality assessment methods and measures, with particular attention to astronomic photographs; we also review most used contrast measures. In paragraph 3 we briefly review SCA algorithms and recall the principles of ACE (Automatic Color Equalization) algorithm as well as CLAHE (Contrast Limited Adaptive Histogram Equalization) algorithm. In paragraph 4 we resume the characteristics of the images we have selected to perform comparisons. The comparison has been made with two algorithms, ACE and the local contrast algorithm used in some astro-photographs processing program CLAHE. In paragraph 5 we present the results of measurements and discuss their effectiveness. In paragraph 6 a conclusion is drawn.

\section{QUALITATIVE AND QUANTITATIVE EVALUATION OF ASTRONOMIC PHOTOGRAPHS}

Differently from natural terrestrial scenes or portrait photographs, astronomy photographs reproduce subject that are characterized by specific features. First the stars are point wise subjects, their observation and capture are influenced by diffraction that depends on the aperture of the device. The kind of sky subjects range from stars to planets and satellites, and from galaxies to nebulae. The quality of all these subjects observation still depends on the diffraction limits but also contrast plays a relevant role. The range of luminance that can be observed in a single photograph is quite high. In natural photography the luminance ranges between 1 to $\approx 1.000 .000 \mathrm{~cd} / \mathrm{m} 2$, while astronomy photographs taken from earth with amateur telescopes can range from magnitude -1 to magnitude 12 , corresponding to a ratio $\approx 1: 400.000$ (we do not consider the problem of astronomical seeing, assuming the best possible conditions). Special filters can also be used to extend the spectral range beyond the visual interval spectrum.

We deal with high dynamic range images, and the rendering problem is to find an effective tone mapping for monitor display or print. To render astro-photographs it is important to have the best possible contrast before finding a tone mapping solution, without recurring to specific HDR methods.

\subsection{Image quality assessment}

Image quality assessment is based normally on five attributes ${ }^{1}$ : yone (or global contrast), color, resolution, sharpness and noise. Astronomic photographs are mainly affected by noise induced by the sensor devices. It can be reduced by specific algorithms, using if necessary dark field, flat field and bias snapshots. Sharpness is an important attribute in particular for planetary, sun and moon photographs; it is usually increased by applying unsharp masking method. For deep sky photographs sharpness is not so important, and unsharp masking can also introduce relevant artifacts. Resolution is also an important attribute, but it is limited by the diffraction properties of the equipment (optics, sensor), and by the seeing conditions. Color should also be an important feature but there are two aspects to be considered. First of all our visual system has not evolved to perceive star, planets and deep sky object colors, therefore we do not know which would be the "right" color. Moreover we are primarily faced with a specific requirement: colors are used to emphasize physical attributes of the subject, particularly in nebulae and galaxies. Orbiting telescopes use special filters to detect information related to the nature of gas (emitting or reflecting) or the nature of planetary soil. Therefore color reproduction can be considered as a false color processing. From this viewpoint color contrast can be an important attribute.

On the other hand there are some artifacts particularly distasteful. Contouring is due to a poor quantization. Jaggedness or pixelization is caused by insufficient space resolution. Aliasing relates to inadequate signal sampling. Blocking is typical of poor DCT compressed images; smudging and color bleeding are due to DWT compression (we note that blocking and smudging should not be important, being related to image compression, while the processing is performed 
on raw or RGB/LRGB non compressed color images). Ringing is caused by inappropriate frequency based filtering. Halo derives from an excess of digital sharpening around edges (similar to ringing). Patterning is an effect induced by dithering. Digital flare originates by stray light coming from areas or point sources, typical of star images. Other artifacts like streaking, banding and color mis-registration can be present in printed reproduction. We note that, in many cases, the relationship of local and global contrast is not sufficiently investigated as a quality attribute for astro photographs.

When rendering astro photographs attributes and artifacts are to be seriously considered to assess the image quality. Image quality assessment is influenced also by the subject or scene content. For instance a poor sharpened human portrait can be considered pleasant or even excellent from an artistic viewpoint, while an image containing a fine pattern like a brick wall is considered as poor or bad if it is blurred. This situation is very important in astro photographs, in particular planets, moon or sun are considered good or excellent when fine details can be observed, while a nebula picture can be judged as good or excellent even if the blurring is relatively strong. On the other hand ringing or halo can be considered annoying.

Some of the above image qualities can be measured or assessed by subjective visual evaluation. In particular we compute measures to assess noise and contrast, leaving to a subjective judgment the global evaluation of the resulting images.

\subsection{Contrast in photography}

A unique and agreed definition of visual contrast in photography does not exist. It is usual to consider contrast in natural photography as a well-balanced distribution of light and dark tones. In some cases an excess of light tones or dark tones is specifically desired by the photographer to get high-key or low-key pictures. In this context the contrast is a poetic aspect of the picture, revealing or not visual details. In image processing a well-balanced tone distribution is measured with the help of the histogram. As the histogram is more flat, the picture has better distributed tones. It could seem effective to improve the contrast by globally correcting the histogram with the e.g. the Global Histogram Equalization method, but this is not suited for astro photographs, since the large number of very dark pixels correspond to a high peak in the histogram and the method gives rise to insufficient range levels for the most important light pixels. Moreover in photography we meet also color contrast besides light and dark tone distribution, which is another expressive aspect of photography.

From a more technical viewpoint another aspect of contrast deals with the photographic material used to reproduce the exposure range: the sensitometric contrast, which in analog printed photographs is measured by densitometry devices. It is known in analog photography that insufficient exposure produce a lower contrast. To deal with these two aspects a tone mapping function is used.

A widely diffused measure of global contrast is the Michelson contrast measure $\frac{L_{\max }-L_{\min }}{L_{\max }+L_{\min }}$ where $L_{\max }$ and $L_{\min }$ are the maximum and minimum luminance. This measure is far from the visual contrast, where the local distribution of tones is of primary importance and gives a very rough description of the characteristics of the photograph that is particularly critical in astronomic photographs, as we will investigate in this paper.

\subsection{Improving contrast}

To improve the contrast without using global histogram equalization a number of solutions have been proposed in image processing research. A brief review is given here.

Beghdadi et al. ${ }^{2}$ use local contrast methods inspired by Gordon et al. ${ }^{3}$ where locality is found by edge detection in a neighborhood of pixel in textured areas, and amplifying the difference between a pixel and its local surround. Since this method increases noise, they move from a proposal of a local contrast measure based first on the detection of regions delimited by edges, then associating to each area a contrast measure $C_{k, l}=\frac{\left|X_{k l}-E_{k, l}\right|}{\left|X_{k l}+E_{k, l}\right|}$ where $C_{k l}$ is the contrast 
associated to pixel $(k, l)$ and $E_{k l}$ is the mean edge grey level, defined as $E_{k, l}=\frac{\sum_{(i, j) \in N(k, l)} G_{i, j} * X_{i, j}}{\sum_{(i, j) \in N(k, l)} G_{i, j}}$ where $G_{i, j}$ is the pixel value at the edge. The computed local contrast is transformed with a simple function depending on being local contrast larger or smaller than the associated edge mean value: $X_{k, l}^{\prime}=E_{k, l} \frac{1-C_{k, l}}{1+C_{k, l}}$ if $X_{k, l} \leq E_{k, l}$ or $X_{k, l}^{\prime}=E_{k, l} \frac{1+C_{k, l}}{1-C_{k, l}}$ if $X_{k, l}>E_{k, l}$. This method is not particularly suited to astro photographs, since the noise increase is still relevant.

Celik et al. ${ }^{4}$ extend the method proposed by Gordon et al. ${ }^{5}$ creating a linear mixture of Gaussian distributions to partition the grey levels into regions that are separately equalized computing the respective cumulative distribution functions. In this way each grey level histogram is mapped separately adjusting brightness and keeping the variance of the grey levels. To evaluate the results they use different metrics: Gordon's local contrast measure, absolute mean brightness error proposed by Chen et al. ${ }^{6}$, and discrete entropy.

Stark $^{7}$ proposes an adaptive histogram equalization that is based on considering small windows of the image, computing locally a cumulation function to create mapping into output grey levels. To improve the method a Gaussian blur of the local histogram is applied. The method can have too strong effects and is very sensitive to the cumulation function variants.

Garg et al. ${ }^{8}$, Neethu et al. ${ }^{9}$ adopt contrast limited adaptive histogram equalization (CLAHE). This method has been devised in the context of medical imaging. CLAHE is based on a tiling of the image, equalization of each tile limiting the local contrast to minimize noise amplification and final stitching with a bilinear interpolation. The final interpolation as well as tile extension can influence the final result. In some implementation the final image is obtained by a weighted average of original and post-processed images.

Beside automatic contrast enhancement method a widely used approach in astro photography is an interactive manipulation of mapping curves and histogram stretching, operating with photographic image processing programs like Photoshop or GIMP. Also specific astronomic image processing programs like MaximDL, IRIS, PixInsight offer interactive approach to improve contrast of astro images.

The above algorithms are mainly derived from research in medical imaging and it is important to underline the difference between astronomical images and medical ones. Medical images have structural elements that are absent in galaxies and nebulae pictures. Moreover researchers know the structural elements (vessels, bones, muscles and other tissues) and criteria to verify the correctness of image segmentation do exist based on the ability to separate natural structures to pathological ones, contrary to astronomical images where the subject is totally unknown. As a consequence our major criticism to the methods based on images subdivision or segmentation is the lack of a criterion to subdivide by tiling or edge detection the image. For this reason in particular the method CLAHE, implemented in the astronomical image processing software PixInsight, is highly sensitive to control parameters. On the other hand interactive approaches are time consuming and lack of repeatability. In our method the control of the balance between local and global contrast is driven by human vision principles.

\section{SPATIAL COLOR ALGORITHMS AND CLAHE}

Spatial Color Algorithms (SCAs) belong to a family of computational models of human visual system, which aim to estimate the color appearance of an observed scene. For our vision system, different color sensations can be generated by the same levels of radiance, according to the content of the rest of the image. SCAs reproduce this behavior by performing spatial comparisons among the pixels of the input image. Many SCAs exist, derived from Retinex theory by Land and McCann ${ }^{15}$ and Land ${ }^{16}$. Among the many SCA algorithms we recall in particular RSR by Provenzi et al. ${ }^{17}$, 
STRESS by Kolås et al. ${ }^{18}$, ACE by Rizzi et al. ${ }^{19}$, RACE by Provenzi et al. ${ }^{20}$, Termite Retinex by Simone et al. ${ }^{21}$ and QBRIX by Gianini et al. ${ }^{22}$.

\subsection{ACE}

Among the many algorithms belonging to the Retinex family, we have decided to work with ACE (Automatic Color Equalization). The choice is due to the particular method used to compare the target pixel with the others in the image. In fact, the major part of other SCAs use a statistic sampling, that could increase the noise in the image, inappropriate for this kind of images, while ACE compare the target pixel with all the others, without introducing noise.

ACE is composed by two stages. In the first stage, every pixel in the image is compared through arithmetical difference to every other pixel. A non-linear function, used to control the contrast in the final image, is applied to this operation, and every comparison is weighted by a function based on the distance between the two considered pixels.

The second stage is needed to scale the obtained result into the available output dynamic range.

Ace is able to adjust the local and global contrast, processing the three RGB channels separately. One of the advantages of using ACE, is that it is almost unsupervised. There are essentially two parameters to be tuned: the first is slope that has influence on the non-linearity of the contrast; the second is alpha that controls the local/global effect. In practical use, alpha has a very limited visual effect, reducing in practice ACE behavior under the control of just the contrast parameter (slope). To better understand the role of the two parameters we recall that ACE is a two stages process. The first stage is a merge of gray world and white patch approaches and performs a sort of lateral inhibition mechanism weighted by pixel distance (the weight is the alpha parameter). This stage is responsible of the local/global contrast improvement. The second stage maximizes the dynamic range of the image, normalizing the white at a global level only. The parameter slope controls the slope of the linear part of the mapping function. Typical values used for the images processed for this work are alpha $=0.01 \approx 0.09$ and slope $=1.5 \approx 2.5$. Higher values of slope correspond to higher global contrast, while higher values of alpha correspond to higher local contrast.

\subsection{CLAHE}

We already have described the principles of CLAHE algorithm. Its implementation in PixInsight depends on three parameters. CLAHE is designed to be applied after histogram stretching, and computes histogram equalization on neighborhood of pixels. An important difference to ACE is that CLAHE is computed on the luminance channel only. The size of the neighborhood is controlled with the kernel parameter and in general acceptable size is between 32 and 128 pixels. Too small values produce ringing and halo effects. The second parameter is the contrast limit that determines the maximum slope of the transfer function applied to individual pixels. A value 1.0 means no change, as the value increases the effect is very similar to a global histogram equalization, reasonable values are between 1.5 and 3.0. The third parameter is the amount of blending of the original and transformed picture, and ranges from 0.0 to 1.0 , where 0.75 for instance means $1 / 4$ of the original image a $3 / 4$ of the equalized image. The value 1 produces an excess of artifacts. In our experiments we found best results with 128 as the kernel size, 3.0 as slope and 0.5 as amount of blending.

\section{MATERIALS AND METHODS}

The subject we have used for our analysis are amateur photographs taken with a reflector telescope SkyWatcher 150/750 and using a photographic camera Fujifilm X-E1 with the X-Trans sensor with $4896 \times 3264,4.8 \mu$ pixels. The subject is the galaxy M31, Andromeda. In a previous work ${ }^{10}$ we have compared the approach also with photographs downloaded from sky survey repository of different subjects, including nebulae and star clusters.

The photographs that we have analyzed are three series of raw snapshots, 12 bits depth. The series were shot at 1600 ISO with changing exposure times: 120 ", 240", 360". Considering the telescope mount (Celestron EQ5) we decided to limit the exposure time to 6', to avoid motion effects. Each series comprises also 5 dark fields, 20 flat fields and one series of 100 bias fields.

We have decided to compare results for different exposure times of the same subject for two reasons: first the increase of the exposure time produce an increase in sensitometric contrast so that we expect to observe an increase in contrast measure before and after post processing as exposure time increase. The second reason is that higher exposure times 
increase also the noise since the camera is not cooled. As a consequence we can better confirm and quantify the effect of the contrast enhancement methods to the noise level of the final image.

The photographs have been calibrated and stacked with PixInsight ${ }^{11}$, after a $4 \times 4$ subsampling to reduce artifacts related to oversampling of the sensor with respect to the diffraction limit of the telescope. After calibration and stacking the Dynamic Background Extraction process of PixInsight has also been applied to improve the quality of the background sky. The resulting three images have been saved in .fits format and processed with FitsLiberator ${ }^{12}$ to de-linearize the histogram mapping. To this aim we have used the arcSinh function. The resulting three channel images have been imported into Photoshop and converted into a RGB color image. Since the camera characteristics generate a strong red dominant we have also applied the automatic color balancing tool of Photoshop. The resulting image has been saved in .tiff format and the contrast enhancement algorithms ACE and CLAHE have been applied.

On the post-processed images we have applied some measures to verify the contrast improvement, and the amount of noise increase induced by the chosen algorithms.

The computed measures are:

- Noise level. The noise level estimation algorithm is based on multi-resolution support obtained by wavelet decomposition ${ }^{13}$, computed with PixInsigth.

- $\quad$ RMS, computed with PixInsigth.

- Entropy, computed with Matlab.

- Global contrast, which is Michelson contrast averaged across blocks of 10x10 pixels, computed with Matlab.

- Multilevel contrast has been proposed by Rizzi et al. ${ }^{14}$, it measures the local contrast.

- $\quad$ PSNR measures the noise introduced by the contrast enhancement method with respect to the original image, it is computed with Matlab.

We have computed PSNR considering the image before post-processing as the reference image, to assess the acceptability of the result as is usual in other image processing field, e.g. image compression.

\section{RESULTS}

We have measured parameters of the original image after calibration, alignment, integration, noise reduction and background removal and before post-processing. Then we made the same measures after post processing with ACE and CLAHE methods. We recall that post processing is applied after stretching the histogram by a arcSinh mapping function. Moreover the measures have also been computed for the luminance channel L extracted from the RGB images. In table 1 the results of the first series of images and in table 2 the same measures on the luminance channel L only.

Table 1. Measures on color images

\begin{tabular}{|l|l|l|l|l|l|l|l|l|l|}
\hline $\begin{array}{l}\text { Exposure } \\
\text { time }\end{array}$ & \multicolumn{3}{|c|}{ 120” } & \multicolumn{3}{c|}{ 240” } & \multicolumn{3}{c|}{$36 \%$} \\
\hline Measure & $\begin{array}{c}\text { Before } \\
\text { post-proc }\end{array}$ & ACE & CLAHE & $\begin{array}{c}\text { Before } \\
\text { post-proc }\end{array}$ & ACE & CLAHE & $\begin{array}{c}\text { Before } \\
\text { post-proc }\end{array}$ & ACE & CLAHE \\
\hline $\begin{array}{l}\text { Noise } \\
\text { evel }\end{array}$ & $4,92 \mathrm{E}-05$ & $3,35 \mathrm{E}-02$ & $1,31 \mathrm{E}-02$ & $7,55 \mathrm{E}-05$ & $1,12 \mathrm{E}-02$ & $1,28 \mathrm{E}-03$ & $2,99 \mathrm{E}-04$ & $2,91 \mathrm{E}-02$ & $2,52 \mathrm{E}-02$ \\
\hline Entropy & $4,78 \mathrm{E}-01$ & $5,36 \mathrm{E}+00$ & $6,19 \mathrm{E}+00$ & $1,22 \mathrm{E}+00$ & $5,85 \mathrm{E}+00$ & $6,14 \mathrm{E}+00$ & $9,26 \mathrm{E}-01$ & $6,63 \mathrm{E}+00$ & $6,68 \mathrm{E}+00$ \\
\hline $\begin{array}{l}\text { Global } \\
\text { contrast }\end{array}$ & $9,68 \mathrm{E}-01$ & $9,62 \mathrm{E}-01$ & $9,65 \mathrm{E}-01$ & $9,59 \mathrm{E}-01$ & $9,14 \mathrm{E}-01$ & $8,78 \mathrm{E}-01$ & $9,96 \mathrm{E}-01$ & $9,71 \mathrm{E}-01$ & $9,60 \mathrm{E}-01$ \\
\hline RMS & $8,50 \mathrm{E}-03$ & $2,24 \mathrm{E}-01$ & $2,98 \mathrm{E}-01$ & $9,95 \mathrm{E}-02$ & $1,11 \mathrm{E}-02$ & $1,06 \mathrm{E}-02$ & $2,89 \mathrm{E}-04$ & $2,01 \mathrm{E}-02$ & $1,68 \mathrm{E}-02$ \\
\hline $\begin{array}{l}\text { Multilevel } \\
\text { contrast }\end{array}$ & $1,41 \mathrm{E}-03$ & $4,29 \mathrm{E}-02$ & $7,30 \mathrm{E}-02$ & $2,49 \mathrm{E}-03$ & $6,83 \mathrm{E}-02$ & $6,92 \mathrm{E}-02$ & $4,17 \mathrm{E}-03$ & $9,88 \mathrm{E}-02$ & $8,47 \mathrm{E}-02$ \\
\hline PSNR & N/A & 29,78 & 27,92 & N/A & 15,37 & 13,81 & N/A & 13,27 & 11,70 \\
\hline
\end{tabular}


Table 2. Measures on Luminance channel

\begin{tabular}{|c|c|c|c|c|c|c|c|c|c|}
\hline Exposure & \multicolumn{3}{|c|}{$120 "$} & \multicolumn{3}{|c|}{$240 "$} & \multicolumn{3}{|c|}{$360 "$} \\
\hline Measure & $\begin{array}{c}\text { Before } \\
\text { post-proc }\end{array}$ & $\mathbf{A C E}$ & CLAHE & $\begin{array}{c}\text { Before } \\
\text { post-proc }\end{array}$ & $\mathrm{ACE}$ & CLAHE & $\begin{array}{c}\text { Before } \\
\text { post-proc }\end{array}$ & $\mathrm{ACE}$ & CLAHE \\
\hline $\begin{array}{l}\text { Noise } \\
\text { level }\end{array}$ & $7,26 \mathrm{E}-06$ & $8,39 \mathrm{E}-03$ & $1,29 \mathrm{E}-02$ & $4,00 \mathrm{E}-06$ & $2,91 \mathrm{E}-03$ & $2,88 \mathrm{E}-03$ & $1,33 \mathrm{E}-04$ & $1,67 \mathrm{E}-02$ & $2,33 \mathrm{E}-02$ \\
\hline Entropy & $2,69 \mathrm{E}-01$ & $5,29 \mathrm{E}+00$ & $6,36 \mathrm{E}+00$ & $1,13 \mathrm{E}+00$ & $5,93 \mathrm{E}+00$ & $6,47 \mathrm{E}+00$ & $1,43 \mathrm{E}+00$ & $6,69 \mathrm{E}+00$ & $6,79 \mathrm{E}+00$ \\
\hline $\begin{array}{l}\text { Global } \\
\text { contrast }\end{array}$ & $1,00 \mathrm{E}+00$ & $9,28 \mathrm{E}-01$ & $9,11 \mathrm{E}-01$ & $1,00 \mathrm{E}+00$ & $9,54 \mathrm{E}-01$ & $9,19 \mathrm{E}-01$ & $9,96 \mathrm{E}-01$ & 9,49E-01 & $9,23 \mathrm{E}-01$ \\
\hline RMS & $8,51 \mathrm{E}-03$ & $8,00 \mathrm{E}-02$ & $8,00 \mathrm{E}-02$ & $4,26 \mathrm{E}-04$ & 1,49E-02 & $1,48 \mathrm{E}-02$ & $3,06 \mathrm{E}-04$ & $2,30 \mathrm{E}-02$ & $1,89 \mathrm{E}-02$ \\
\hline $\begin{array}{l}\text { Multilevel } \\
\text { contrast }\end{array}$ & $4,94 \mathrm{E}-04$ & $4,88 \mathrm{E}-02$ & $8,11 \mathrm{E}-02$ & $1,36 \mathrm{E}-03$ & $8,21 \mathrm{E}-02$ & $8,44 \mathrm{E}-02$ & $3,56 \mathrm{E}-03$ & $1,08 \mathrm{E}-01$ & $9,16 \mathrm{E}-02$ \\
\hline PSNR & $\mathrm{N} / \mathrm{A}$ & 26,52490 & 23,35810 & N/A & 16,10 & 14,15 & $\mathrm{~N} / \mathrm{A}$ & 35,12 & 35,13 \\
\hline
\end{tabular}

Let's examine in detail the different measures, and to better appreciate the values we plot the measures for the three exposures.
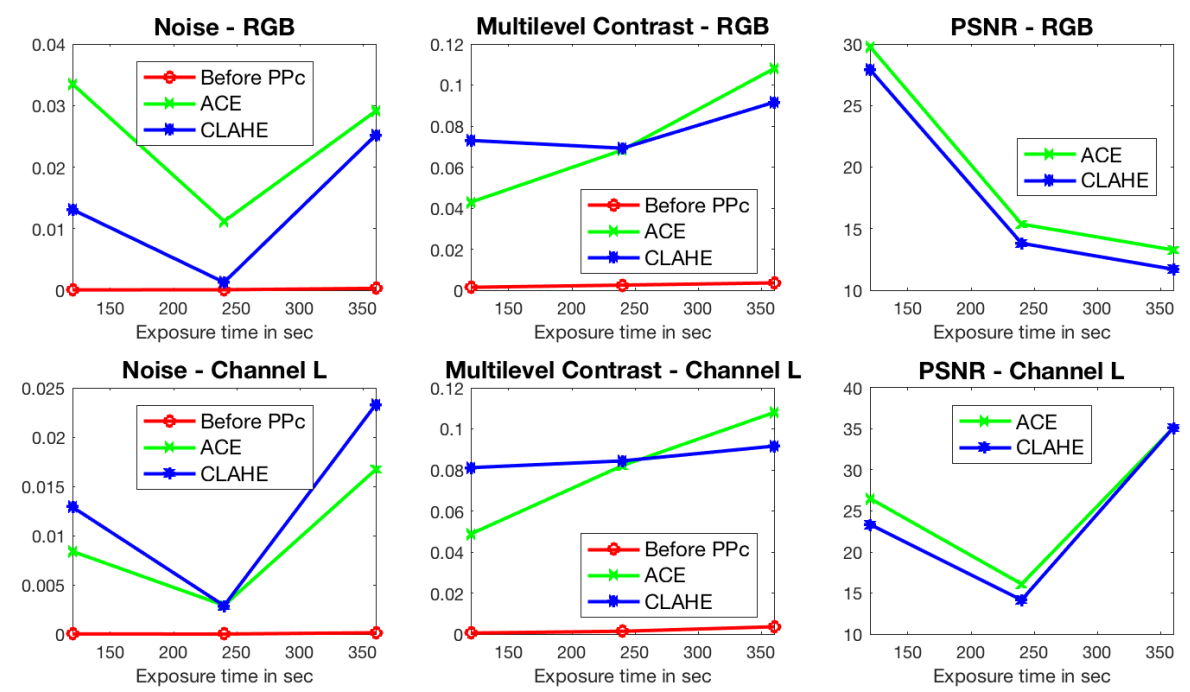

Figure 1. Noise, multilevel contrast, PSNR for color (top) and luminance channel (bottom).

First of all we observe (see Figure 1) that, as expected, the noise level increases with the exposure time. To better understand how the noise behaves we recall that the noise level of the raw images before any pre-processing ranges in the interval $[2.64 \mathrm{E}-04 \approx 3.91 \mathrm{E}-04]$ and for the $\mathrm{L}$ channel in the range [1.14E-04 $\approx 1.73 \mathrm{E}-04]$.

After all calibration and integration, as expected, the noise level decreases and after post-processing for contrast enhancement we have an increase of 2 orders of magnitude. ACE has a slightly higher noise increase than CLAHE, due to the fact that the weighted average of the original and post processed images in CLAHE has been done as $50 \%$ each, so the contribution of CLAHE on noise is more limited.

About contrast we note that ACE has a better multilevel contrast than CLAHE for RGB images, while for the luminance channel it is the contrary. This is due to the specific character of ACE that enhances the three channels separately, while 
that region detection in CLAHE is performed on L channel only whose contribution to the three channel is therefore the same.
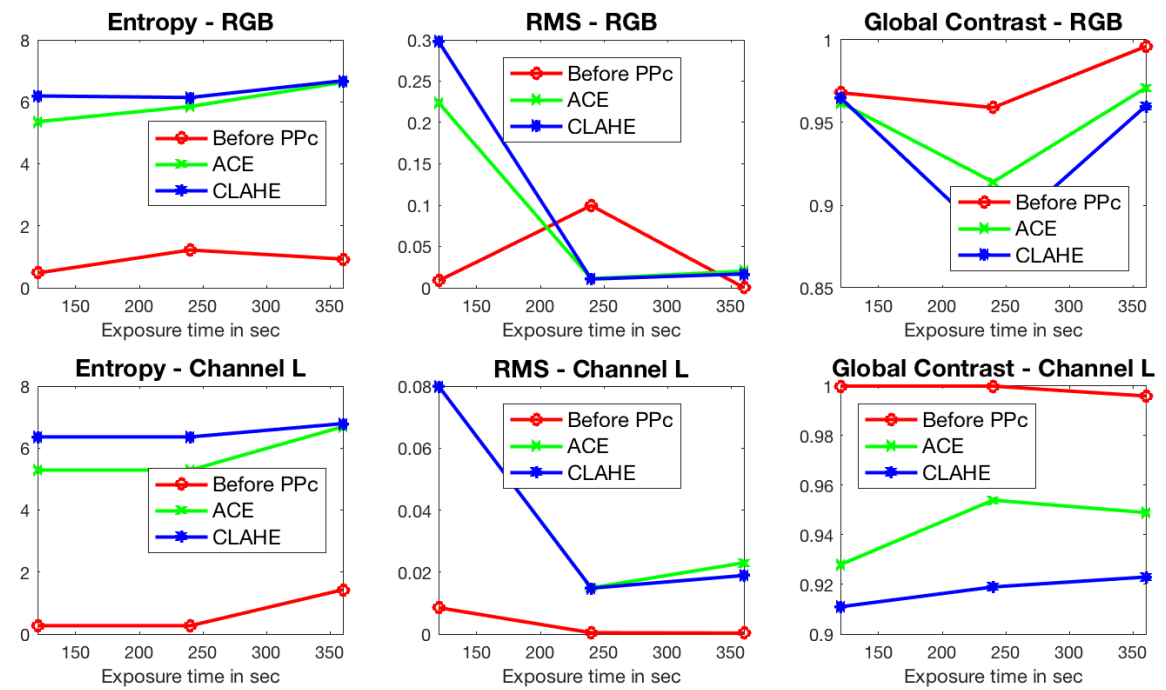

Figure 2. Entropy, RMS and global contrast (top) and luminance channel (bottom)

On the other hand global contrast and RMS (see Figure 2) do not allow to discriminate clearly the different behavior of the two post processing algorithms. In particular global contrast on L channel images has a opposite behavior to multilevel contrast and RMS. Global and multilevel contrast for RGB images evaluate better ACE than CLAHE.

About entropy we observe higher values for both RGB and L channel and for post-processed images. This parameter does not to draw any other specific conclusion.

About PSNR we observe that ACE has a lower PSNR than CLAHE, for 360" images the PSNR has the same value for both.

In figures 3, 4 and 5 we show (from left to right) the un-processed images, the pre-processed images and post-processed images using ACE and CLAHE for the different exposure times. For display purposes the two leftmost images images are shown after histogram stretching, while during processing the histogram is kept linear.

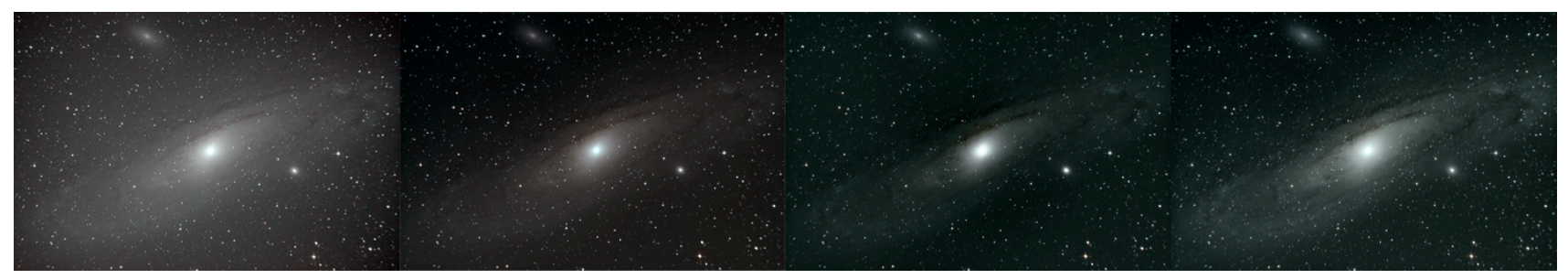

Figure 3 - M31 120" exposure

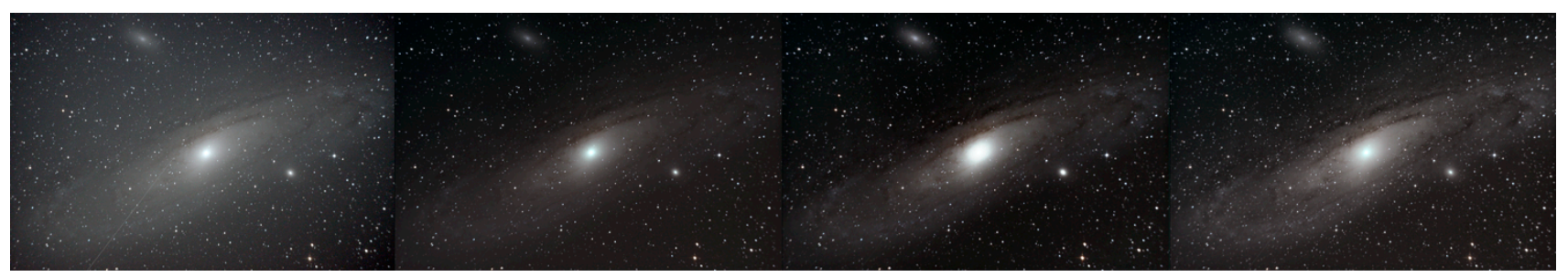

Figure 4 - M31 240" exposure 


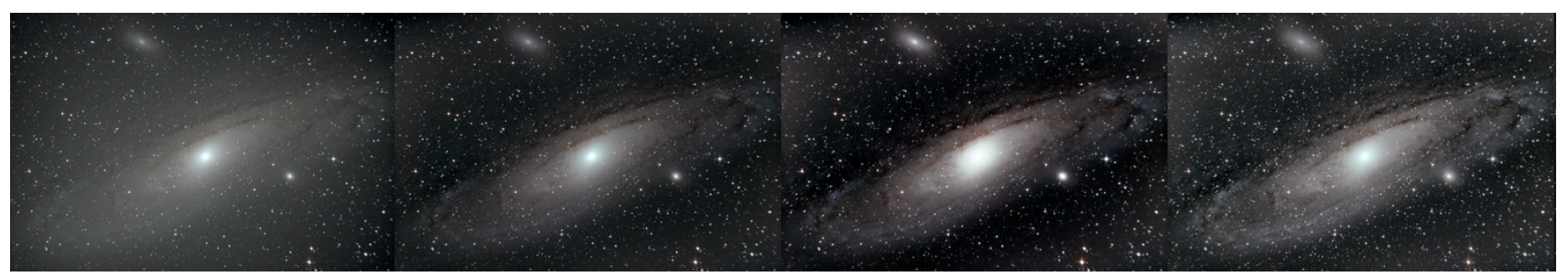

Figure 5 - M31 360” exposure

\section{CONCLUSIONS}

We have compared two algorithms, ACE and CLAHE, for improving the contrast of deep sky galaxy photographs with different exposure times. The algorithm ACE is based on human visual system principles, while CLAHE improves contrast by first subdividing the image in the Luminance channel and using the subdivision to improve locally the image and finally weighted averaging the original and improved images.

By visualy inspecting the resulting images we note that CLAHE is better than ACE for 120" exposure, both algorithm produce very similar images for 240" exposure and ACE outperforms CLAHE for 360".

The measure of multilevel contrast on RGB and channel L only confirms the observation. On the other hand ACE shows a better PSNR to the original image.

The behavior of the noise is coherent on one side to the increase in exposure time, while we observe that both algorithms introduce a noise increase with a better behavior for CLAHE than ACE.

We can conclude that both algorithm can be effective, while we note that both need to interactively tune their parameters, two ACE has and three in CLAHE thus requiring more trial and error.

Using either algorithm is anyway an improvement with respect to interactive manipulation with photo processing software.

\section{REFERENCES}

[1] Allen E., Triantaphillidou S., [The Manual of Photography], $10^{\text {th }}$ Edition, Focal Press, New York \& London, (2009)

[2] Beghdadi A., Le Negrate A., "Contrast Enhancement Technique Based on Local Detection of Edges" Computer Vision, Graphics and Image Processing 46, 162-174 (1989).

[3] Gordon R., Rangayan R.M., "Feature enhancement of film mammograms using fixed and adaptive neighborhoods" Applied Optics 23(4), 560-564 (1984).

[4] Celik T., Tjahjadi T., "Automatic Image Equalization and Contrast Enhancement Using Gaussian Mixture Modeling” IEEE TIP 21(1) 145-156 (2012).

[5] Gordon R., Rangaraj M.R., "Feature enhancement of film mammograms using fixed and adaptive neighborhoods" Applied Optics 23, 4, 560-564 (1984).

[6] Chen S.-D., Ramli A., "Minimum mean brightness error bi-histogram equalization in contrast enhancement" IEEE Trans. Consum. Electron. 49 (4), 1310-1319 (2003).

[7] Stark J. A., “Adaptive Image Contrast Enhancement Using Generalizations of Histogram Equalization,” IEEE TIP 9(5), 889-896 (2000).

[8] Garg R., Mittal B., Garg S., "Histogram Equalization Techniques for Image Enhancement” IJECT 2 (1), $107-$ $111(2011)$ 
[9] Neethu M. Sasi, V. K. Jayasree, "Contrast Limited Adaptive Histogram Equalization for Qualitative

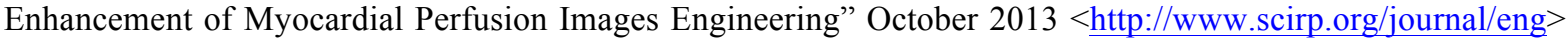
accessed March, (2016).

[10] Marini D.L.R., Bonanomi C., Rizzi A., "Processing astro-photographs using Retinex based methods" Proceedings IS\&T Electronic Imaging EI2016, 023.1-10 (2016).

[11] https://pixinsight.com, accessed April (2016).

[12] https://www.spacetelescope.org/projects/fits liberator/, accessed April (2016).

[13] Starck JL. Murtagh F., [Astronomical Image and Data Analysis], Springer, 36-39 (2002).

[14] Rizzi A., Algeri, T., Medeghini G., Marini D., "A proposal for contrast measure in digital images" Proc. CGIV04, Berlin, IS\&T, 187-191 (2004).

[15] Land E. H., McCann J. J., "Lightness and the retinex theory" J.Opt.Soc.Am 63, 1-11 (1971).

[16] Land E. H., "The Retinex Theory of Color Vision” Scientific American 237, 6, 108-128 (1977).

[17] Provenzi E., Fierro M., Rizzi A., DeCarli L., Gadia D., Marini D.L.R., "Random Spray Retinex: a new Retinex implementation to investigate the local properties of the model" IEEE Transactions on Image Processing 16, 1, 162-171 (2007).

[18] Kolås O., Farup I., Rizzi A., "STRESS: A Framework for Spatial Color Algorithms” Journal of Imaging Science and Technology 55, 4, 040503-1-040503-10 (2011).

[19] Rizzi A., Gatta C., Marini D.L.R., “A new algorithm for unsupervised global and local color correction” Pattern Recognition Letters 24, 663- 1677 (2003).

[20] Provenzi E., Gatta C., Fierro M., Rizzi A., "A spatially variant white patch and gray world method for color image enhancement driven by local contrast" IEEE Transactions on Pattern Analysis and Machine Intelligence 30, 10, 1757-1770 (2008).

[21] Simone G., Audino G., Farup I., Albregtsen F., Rizzi A., "Termite retinex: A new implementation based on a colony of intelligent agents" Journal of Electronic Imaging 23, 1, 013006-013006 (2014).

[22] Gianini G., Manenti A., Rizzi A., "Qbrix: a quantile-based approach to retinex" Journal of the Optical Society of America 31, 12, 2663- 2673 (2014). 